\title{
Disaster management - Issues for action
}

Major emergencies and disasters, natural or chemical releases, have occurred across the globe and as the population grows and resources become more limited, communities are increasingly vulnerable to the hazards that cause disasters. Emergencies and disasters do not affect only health and well being; frequently large number of people are displaced, killed or injured or subjected to a greater risk of epidemics such as the recent out break of Leptospirosis and Dengue in Mumbai.

A disaster is an occurrence that causes damage, ecological disruption, loss of human life or deterioration of health and health services on a scale sufficient to warrant an extraordinary response from outside the affected community or area. Rapid industrialization and new technologies have produced new hazards. The severity and frequency of technological emergencies have increased. With proliferation of nuclear power and chemical plants over the last few decades, disasters on the scale of Chernobyl or Bhopal cannot be ruled out.

The risks of accidental chemical releases escalate as a number of new hazardous substances are produced. First, production, transport, and use of flammable, explosive or toxic chemicals have grown significantly in both developing and developed countries. Second, greater and more centralized productions have increased the quantities of chemicals manufactured and the distances across which they are transported throughout the country. Third, in a country like ours, population growth nearer to chemical plants and along transportation routes has meant that there are larger communities in great number at high risk following a chemical accident. In India, we have 2,000,000 registered industries, of which more than 5000 are chemical industries. Therefore, it is mandatory for us to have a disaster management plan and the preparedness to avert any loss of life, human suffering, and economic losses like the terrifying event that occurred in Bhopal. Political stability and political will is most needed to drive such initiatives in any country.

With this background let us look at issue for actions with respect to disaster management in our own backyard. A national policy is in place for disaster management but the ownership is lacking and there is no political pressure for total implementation to construct good roads and highways, create facilities for rapid mode of transportation, earmark areas for evacuation of population, networked ambulance services with global positioning system including air ambulance services, radio communication, and intersectorial co-ordination amongst governmental agencies. Lack of action or slow development on the above issues at all levels results into an unprepared society for natural or chemical calamities. There are effective legislations for control of transportation and storage of hazardous chemicals but many smallscale chemical industries causing pollution and threatening to spill off an emergency compound the problem. Lack of manpower in surveillance agencies also increases the risk of dangerous events of chemical release.

Now coming closer to on-site-related issues in hazardous industries, many of them especially small-scale and medium-sized industries have no document of an "On-site disaster management plan" in place and in many large industries it is only on paper. This kind of unpreparedness would result in a serious outcome for the site and also to the society. As prewritten protocol, mock drills are required to be conducted periodically, however, they are not seriously practiced, which contributes to unprepared status and results in poor and chaotic response in the event of an emergency. The concept of "mutual aid" from neighboring industries and community resources that should be a part of the drill never gets tested. The inter-industry interaction and collaboration in discussing emerging scenarios after chemical release, environmental impacts, expectation and roles, and resource pooling are never seriously discussed. The community resource identification both in terms of local and technical experts in various fields and local logistic help groups is poorly done or not done at all. This kind of "community-resource group" is

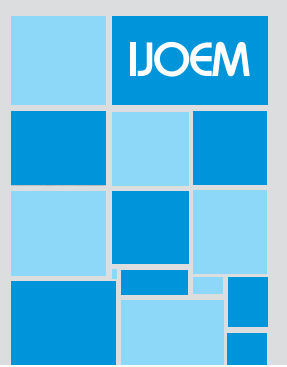


essential for disseminating information on chemical release to the local community to achieve better societal preparedness and to seek better societal co-operation in the event of an emergency. The societal communication in building effective responding ability is an important issue to be addressed by the industry group. When the emergency does not remain focal or local and becomes a regional event then environmental fallouts affecting safe water, food handling, vector control, and sanitation become important issues. Predisaster planning, preventative maintenance, training, and education of people for emergency response services, anticipation of fallouts and continuous planning are only means to overcome such events quickly.

Emerging issues of global threat by terrorists, nuclear proliferation, biological weapons, and chemical warfare pose different kinds of challenges to deal with, which requires global strategy, intelligence sharing, political will and international co-operation on matters of security. This is necessary to prevent such attacks and develop public health and safety response to minimize the impact. This editorial has not only surfaced some of the issues related to disaster management requiring action from various agencies, but also it aims to provide scope for introspection of the current status and some futuristic scenarios that need to be addressed. I am sure that this editorial shall provide an impetus for the members and other connected agencies who shall effectively network on the issues and actions related to disaster management.
G. K. Kulkarni Editor - IJOEM 